\title{
Cleveland Clinic Socre - What is the Role of a Novel Prognostic Treadmil Score to Diagnose Coronary Artery Disease?
}

\author{
MD MASHIUL ALAM, MD MUKHLESUR RAHMAN, TANJIMA PARVIN, KHURSHED AHMED, \\ S M MUSTAFA ZAMAN, SAJAL KRISHNA BANERJEE, SAYED ALI AHSAN, HARISUL HOQUE, \\ PRASHANT BAJRACHARYA, MD RAKIBUL HASAN RASHED
}

Department of Cardiology, University Cardiac Center, Bangabandhu Sheikh Mujib Medical University (BSMMU), Dhaka Address of Correspondence: Dr. Md Mashiul Alam, Resident, Department of Cardiology, BSMMU, Dhaka, E-mail:mashiull@gmail.com

\begin{abstract}
:
Backgroud: Ischemic heart disease is increasing all over the world even in the developing countries like Bangladesh. The incidence rate of coronary artery disease is escalating very rapidly among both male and female population in our country. Though exercise treadmill test (ETT) is a well accepted non-invasive investigation to diagnose Coronary Artery Disease (CAD), it has a high false positive and false negative result if ST segment response alone is calculated for interpretation of the test. Accuracy of different treadmill scores in our population is largely unknown. Clevelan Clinic Score is a prognostic ETT score which is validated for prognostic indication but may have some diagnostic value as well.

Objective: To know the diagnostic role of Cleveland Clinic Score, currently which has only prognostic implication.

Method: A Cross-Sectional study was carried out on patients attending University Cardiac Center in Bangabandhu Sheikh Mujib Medical University (BSMMU) for stable chest pain to find out the accuracy of Cleveland Clinic Scores (CCS) in comparison to other diagnostic treadmill scores namely Duke Treadmill Score (DTS) and Simple Treadmill Score (STS).Total 130 persons including male and female who have undergone ETT were included according to inclusion and exclusion criteria. Coronary angiogram reports were collected after the procedure was performed as per clinical practice. The accuracy of ST segment response \& different treadmill scores were calculated and compared with each other.

Result: ETT scores had better sensitivity and specificity than ST segment response which was affected by workup bias more. CCS, DTS and STS have $83.3 \%$ \& $60.9 \% ; 71.4 \%$ \& $71.7 \% ; 64.3 \%$ \& $78.3 \%$ sensitivity \& specificity, respectively. Receiver Operator Characteristics (ROC) curve analysis showed all of the three scores have similar area under curve (AUC) that means they have similar accuracy to diagnose CAD. But they have different sensitivity and specificity for different cut off value. Overall analysis showed accuracy of STS (83.9\%) is comparable to that of DTS (83.3\%), CCS (77\%) .

Conclusion: Among the three treadmill scores Cleveland Clinic Score has comparable predictive accuracy when compared with DTS, STS. Though a prognostic ETT score, CCS may have diagnostic role which need to be validated further.

Key words: Stable IHD, Coronary artery disease, ETT, Treadmill scores, Duke Treadmill Score, Simple Treadmill Score, Cleveland Clinic Score, Coronary angiogram.
\end{abstract}

University Heart Journal 2018; 14(2): 62-66

Introduction:

Mortality from CAD is increasing in this era of industrialization where sedentary and stressful lifestyle, food adulteration and increased consumption of fatty food have fallen out of scale than previous days. In some areas around the world it has been increased to an epidemic proportion. Recently IHD has emerged as an alarming disease in Central Asia, South Asia and East Asia, leading to an increased burden of treatment cost and disability. CAD is the leading cause of mortality in 
Bangladesh also. Like other South Asians, Bangladeshis are unduly prone to develop $\mathrm{CAD}$, which is often premature in onset, follows a rapidly progressive course and angiographically more severe. As a developing country it has to fight many socioeconomic difficulties, let alone the poor resource that can be provided for health. Allocation provided for cardiovascular disease prevention and treatment is low due to less government expenditure in health. As a result a vast majority of our country people are underprivileged when it comes to costly diagnosis and treatment of coronary artery disease. Non-invasive tests should be utilized and used appropriately in view of this current health condition in our country.

The sensitivity and specificity of ETT varies considerably. According to a meta-analysis conducted by Gianrossi et al. ${ }^{1}$ (1989) there was a wide variability in sensitivity and specificity of ETT [sensitivity $68 \pm 16 \%$ (range $23-$ $100 \%$ ); specificity $77 \pm 17 \%$ (range: $17-100 \%$ ). Another Meta analysis showed sensitivity of $81 \pm 12 \%$ (range: 40 $100 \%$ ) and specificity of $66 \pm 16 \%$ (range: $17-100 \%)^{2}$.

At present several treadmill scores have been proposed as means for improving the diagnostic accuracy of the exercise treadmill test (ETT) and to predict future risk of cardiac. ${ }^{3,4}$ The Duke Treadmill Score (DTS), traditionally a prognostic score, was recently tested as a diagnostic score and shown to predict CAD better than the ST response alone. ${ }^{5}$ Using Simple Treadmill Score has increased accuracy of treadmill test in male and female population, which is more than Duke Treadmill Score in some studies. ${ }^{6,7}$

Cleveland Clinic Score is a prognostic score that was developed by Lauer et al. ${ }^{8}$ in 2007 to predict the survivability of patients presenting with stable chest pain. It also includes many cardiovascular risk factors and variables after exercise treadmill scores. It was validated for risk prediction. To the best of our knowledge none has tested its accuracy to predict coronary artery disease till now.

In our study we compared the diagnostic accuracy of these well known scores namely, Cleveland Clinic Score, Duke Treadmill Score, Simple Treadmill Score to identify significant coronary artery lesion in Bangladeshi male and female patients.

\section{Method:}

We have selected patients whos age was between 30-69 years presented with stable chest pain in Bangabandhu
Sheikh Mujib Medical University and had undergone ETT according to Bruce protocol. Duration of interval between ETT and CAG was arbitarily held 6 months. ${ }^{9}$ Detailed and thorough clinical assessment was done and recorded. All available previous medical documents were checked meticulously. Patients with previous history of myocardial infarction (MI), previous revascularization, already a diagnosed case of CAD were excluded from the study to prevent the falsely elevated accuracy of ETT. Baseline abnormalities that may obscure electrocardiographic changes during exercise e.g., Left bundle branch block or Right bundle branch block, Left ventricular hypertrophy with repolarization abnormality, Digitalis therapy, Vantricular paced rhythm, WolfPerkinson-White syndrome, ST abnormality associated with supraventricular tachycardia or atrial fibrillation were among exlusion criteria as well. Informed written consent was taken from the patient. CAG report was collected from the Cath lab after the procedure.

Visual ST-segment deviation was measured at the J junction and will be corrected for pre-exercise STsegment depression while standing; ST slope was measured over the following $80 \mathrm{~ms}$ and will be classified as horizontal, down sloping and up sloping. Slope was coded as 1 horizontal, down sloping, or upsloping ST depression and 0 for normal slope (up sloping $<2 \mathrm{~mm}$ or ST-segment depression of $<0.5 \mathrm{~mm}$ ). The ST response was considered which has the most horizontal, down sloping or up sloping ST-segment depression in any lead, except aVR, during exercise or recovery. An abnormal response was defined as $\geq 1 \mathrm{~mm}$ of horizontal or downsloping or $\geq 2 \mathrm{~mm}$ upsloping ST-segment depression. ${ }^{10}$ The exercise tests were analyzed, and reported per standard protocol and manual calculation.

Coronary artery narrowing was visually estimated in two orthogonal views by interventional cardiologist with the help of built-in cath lab software and expressed as percent lumen diameter stenosis. Patients with a $70 \%$ narrowing in one or more of the following is considered to have significant angiographic coronary artery disease: the left anterior descending, left circumflex, right coronary arteries or their major branches, or a $50 \%$ narrowing in the left main coronary artery. ${ }^{11}$

Using angiographic evidence of $\mathrm{CAD}$ as the reference, area under the curve (AUC) of Receiver 


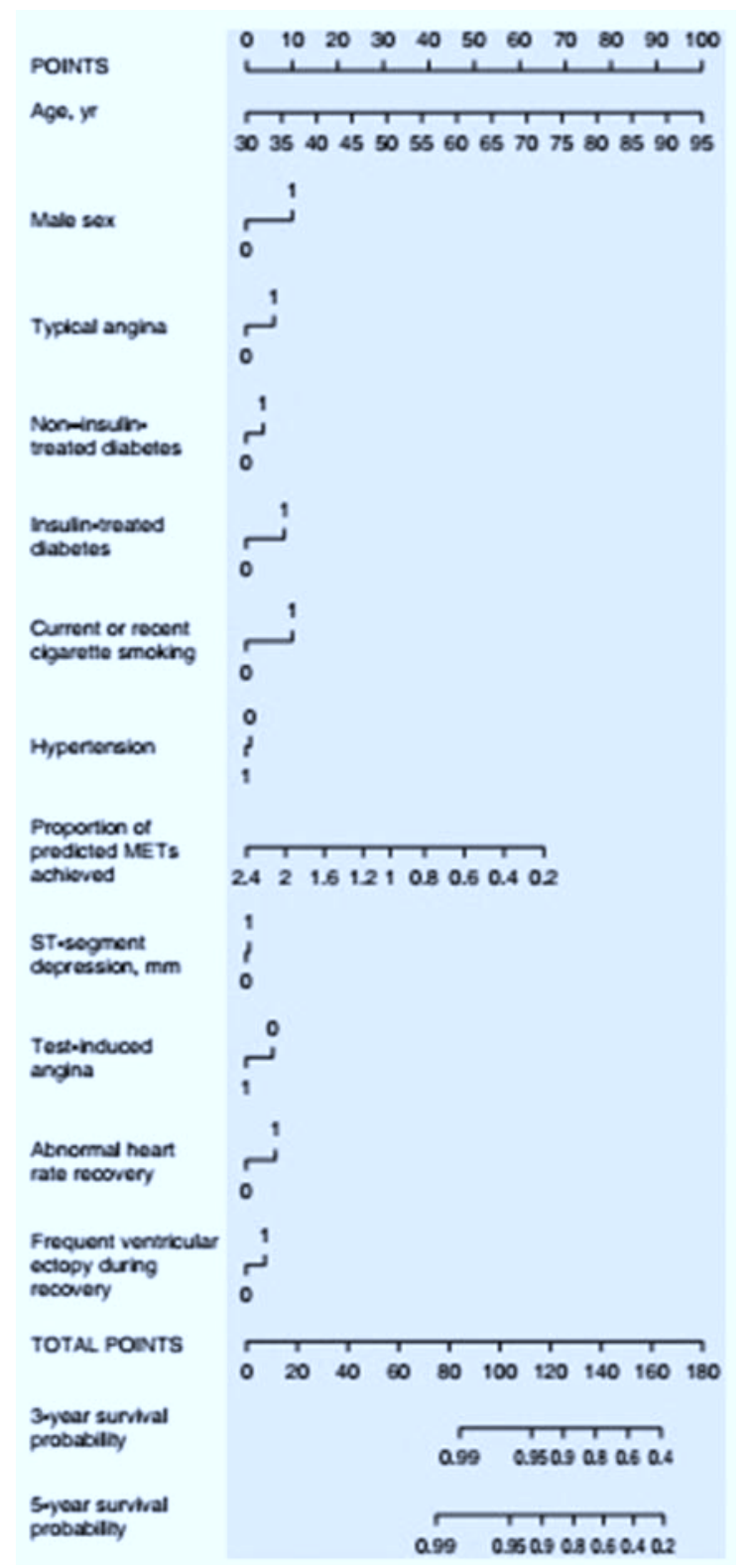

Fig.-1: Cleveland Clinic Nomogram

operating characteristic (ROC) plots were determined for each treadmill score. Statistical analysis was performed with the SPSS and Medcal statistical software.

\section{Result:}

We have taken total 130 male and female patients presented with stable chest pain. Mean age of our sample was $50 \pm 9$ years, whereas mean age among female population is slightly lower than male population (47 \pm $7 \& 51 \pm 9$, respectively). Maximum of the sample were male with male to female ration 2.5:1. 75 (57.7\%) of total sample presented with typical angina, whereas 37 $(28.5 \%) \& 18(13.8 \%)$ were labeled as having atypical $\&$ non-cardiac chest pain, respectively. 70 (53\%) person were smoker, out of them $48(36.9 \%)$ had quit in the past. About half of sample were hypertensive and dyslipidemic; and around one third ofthem were diabetic. $37(18.5 \%)$ people had significant family history of premature CAD.

Table-I

Base line Characteristics.

\begin{tabular}{|c|c|c|}
\hline Variable & $\begin{array}{c}\text { Number } \\
(\mathrm{n}=130)\end{array}$ & $\begin{array}{c}\text { Percentage } \\
(\%) \\
\end{array}$ \\
\hline Mean Age (years) & $50 \pm 9$ & \\
\hline Male & 93 & $71.5 \%$ \\
\hline Female & 37 & $28.5 \%$ \\
\hline \multicolumn{3}{|l|}{ Anginal pain } \\
\hline a) Typical & 75 & $57.7 \%$ \\
\hline b) Atypical & 37 & $28.5 \%$ \\
\hline c) Non-cardiac & 18 & $13.8 \%$ \\
\hline Past smoker & 48 & $36.9 \%$ \\
\hline Current smoker & 22 & $16.9 \%$ \\
\hline $\mathrm{HTN}^{*}$ & 63 & $48.5 \%$ \\
\hline NIDDM* & 36 & $27.7 \%$ \\
\hline IDDM $^{*}$ & 19 & $14.6 \%$ \\
\hline Dyslipidemia & 68 & $57.6 \%$ \\
\hline $\mathrm{FH}$ of $\mathrm{CAD}^{*}$ & 37 & $28.5 \%$ \\
\hline
\end{tabular}

Maximum of the patients having low probability in DTS, STS and CCS had no coronary artery disease. At the same time most of the individuals having high probability score had significant ( $\geq 70 \%$ stenosis) coronary artery disease. DTS and STS had similar accuracy when it is calculated from low and high probability scores and CAG findings (83.3\% \& 83.9\% for DTS \& STS respectively). Accuracy of CCS came around $77 \%$ when it was calculated with similar manner. Chi-square test shows this result is highly significant ( $\mathrm{p}$ value 0.000 ). (Table $-\mathrm{II}$ )

CCS, DTS \& STS have almost similar area under curve (AUC) $(0.771,0.772 \& 0.768$, respectively) in total sample (95\% CI; 0.68-0.84). (Fig-II) There is little difference between the AUC of the scores in pairwise comparison which is not statistically significant ( $p$ $>0.05$ ) (Table -III). AUC of DTS, STS \& CCS has almost similar result in male and female sample separately. 
Table-II

Likelihood of CAD among different ETT scores.

\begin{tabular}{llcccc}
\hline ETT scores & Risk/Probability & CAD & P value & $\begin{array}{c}\text { Accuracy } \\
\text { (TP+TN/ Total } \\
\end{array}$ \\
& & & Present & & $\begin{array}{c}\text { Cases) taking } \\
\text { Low and High } \\
\text { Probability }\end{array}$ \\
\hline CCS & Low & & & $77.2 \%$ \\
& Intermediate & 28 & 14 & 0.000 & \\
\multirow{3}{*}{ STS } & High & 12 & 30 & & $83.9 \%$ \\
& Low & 17 & 40 & & \\
\multirow{5}{*}{ DTS } & Intermediate & 19 & 3 & 0.000 & \\
& High & 10 & 51 & & \\
& Low & 1 & 0 & 0.000 & \\
& Intermediate & 35 & 35 & & \\
& High & 10 & 49 & & \\
\hline
\end{tabular}

Table-III

Pairwise comparison of ROC curves in total sample.

\begin{tabular}{lccc}
\hline Pair & $\begin{array}{c}\text { Difference } \\
\text { between areas }\end{array}$ & $\begin{array}{c}\text { Significance } \\
\text { level }\end{array}$ & SE $^{\mathrm{a}}$ \\
\hline DTS $\sim$ STS & 0.0009 & $\mathrm{p}=0.98$ & 0.04 \\
DTS $\sim$ CCS & 0.0033 & $\mathrm{p}=0.93$ & 0.04 \\
STS $\sim$ CCS & 0.0042 & $\mathrm{p}=0.89$ & 0.03 \\
\hline
\end{tabular}

a DeLong et al., 1988

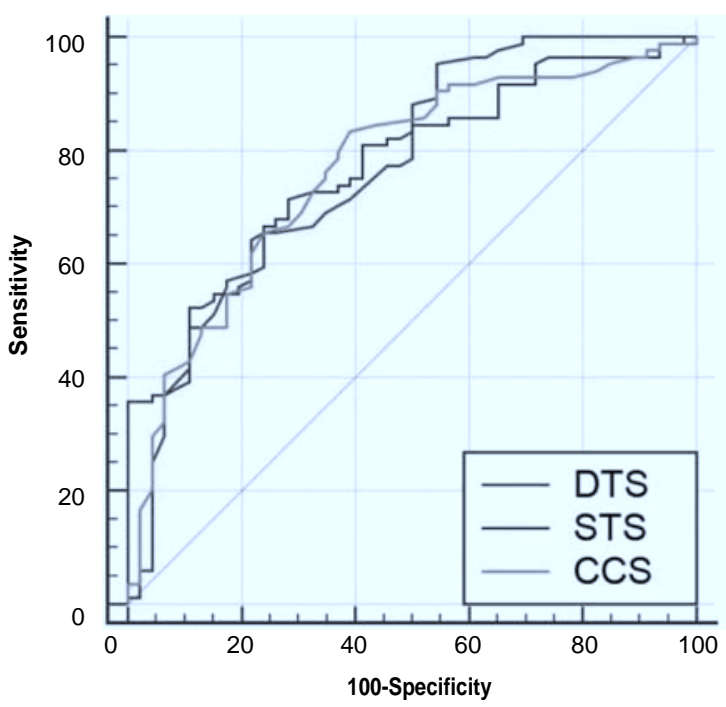

Fig.-2: Comparison of ETT scores in total sample.

\section{Discussion:}

We have investigated 130 patients attending Bangabandhu Sheikh Mujib Medical University (BSMMU) for evaluation of chest pain. All of them had their ETT done recent days or within 6 months by Bruce protocol.
Among 130 individuals male to female ration was 2.5: 1 . Average age of female patients fell in $4^{\text {th }}$ decade (mean age $47 \pm 7$ ), where average age of male sample was $51 \pm 9$ years. The mean age of combined sample population was $50 \pm 9$ years, which differs significantly from the population who undergo ETT in North America (mean age $59.1 \pm 0.3){ }^{9}$

Despite all, our entire sample represents our country population with coronary artery disease. The mean age of people diagnosed as having ischemic heart disease (IHD) in Bangladesh is lower than other developed country as it is true in other South Asian countries. ${ }^{12}$ In 2017 Banerjee et al. showed mean age of people with ischemic heart disease in rural community is $46 \pm 16$ years. ${ }^{13}$

As accuracy of ETT by ST segment response has wide variability different ETT scores were developed over the years namely Duke Treadmill Score (DTS), Simple Treadmill Score (STS) to accurately diagnose CAD by ETT way before invasive and expensive measures. We have analyzed DTS, STS as well as Cleveland Clinic Score (CCS). The latter was tested and validated by Lauer et al. (2007) to predict all cause mortality in adults with suspected coronary artery disease. We have tried to find out whether it can be use as a diagnostic too as it analyze most of the risk factors and more than other diagnostic scores. ${ }^{8}$

CCS has similar accuracy (77\%) in comparison to DTS and STS when we calculated it from low and high probability score; though its accuracy is more than ST segment response. We have arbitrarily used 80 as a cut 
off between low \& intermediate, and 100 as a cut off between intermediate $\&$ high probability. (Fig I)

We have used receiver operating characteristics (ROC) curve to find out cut off value, sensitivity, specificity \& area under curve (AUC) of these three ETT scores. AUC in ROC curve for CCS, DTS \& STS in total sample were $0.768,0.771 \& 0.772$, respectively. The little difference between them are not statistically significant. AUC $>0.7$ -0.8 means all three scores are fair as a diagnostic tool for CAD. More than 0.9 AUC is required to be an excellent test. ${ }^{14}$ Similar AUC of these investigated three scores were found in male and female sample separately.

In summary, we have found CCS, DTS and STS have almost similar accuracy to predict CAD after Exercise treadmill test. Hence, CCS may be utilized as a diagnostic test.

\section{Conclusion:}

Exercise Treadmill Test (ETT) is a well accepted noninvasive tool for prediction of coronary artery disease especially among patients with stable chest pain. It can provide useful information to the clinicians if the test is adequate. Using scoring system in ETT interpretation along with ST segment response increases the predictive accuracy of coronary artery disease. Cleveland Clinic Score is a prognostic score which was tested in our study to identify its role in prediction of CAD. Though it has almost similar accuracy to predict CAD further study is needed for validation of Cleveland Clinic Score as diagnostic score.

\section{Study Limitation:}

Though the study population was larger in comparison to previous studies done on exercise treadmill test in our country, sample size was small to put conclusion on the findings. As many patients were with the positive ETT and underwent coronary angiogram there would have workup bias which could not be eliminated.Our study was a cross-sectional observational study. So some patients cannot be selected by pretest probability, which could affect the findings.

\section{Reference:}

1. Gianrossi R, Detrano R, Mulvihill D, Lehmann K, Dubach P, Colombo A, et al. Exercise-induced ST depression in the diagnosis of coronary artery disease: a metaanalysis. Circulation 1989; 80: 87-98.

2. Myers J, Do D, Herbert W, Ribisl P and Froelicher VF. A nomogram to predict exercise capacity from a specific activity questionnaire and clinical data. Am J Cardiol 1994, 73: 591-96.

3. Berman JL, Wynne J and Cohn PF. A multivariate approach to interpreting treadmill exercise tests in coronary artery disease. Circulation 1978, 58: 505-512.

4. Do D, West JA, Morise A, Atwood E and Froelicher V. A consensus approach to diagnosing coronary artery disease based on clinical and exercise test data. Chest 1997, 111: 1742-49.

5. Shaw LJ, Peterson ED, Shaw LK, Kesler KL, DeLong ER, Harrell FE, Muhlbaier LH, and Mark DB. Use of a Prognostic Treadmill Score in Identifying Diagnostic Coronary Disease Subgroups. Circulation 1998, 98:1622-30.

6. Raxwal V, Shetler K, Morise A, Do D, Myers J, Atwood JE, and Froelicher VF. Simple treadmill score to diagnose coronary disease. Chest 2002 Jun, 119(6):1933-40.

7. Morise AP, Detrano R, Bobbio M, and Diamond GA. Development and validation of a logistic regression-derived algorithm for estimating the incremental probability of coronary artery disease before and after exercise testing. J Am Coll Cardiol 1992, 20: 1187-96.

8. Lauer MS, Pothier CE, Magid DJ, Smith SS and Kattan MW. An externally validated model for predicting long-term survival after exercise treadmill testing in patients with suspected coronary artery disease and a normal electrocardiogram. Ann Intern Med 2007, 147(821): 821-8.

9. Fearon WF, Gauri AJ, Myers J, Raxwal VK, Atwood JE and Froelicher VF. A comparison of treadmill scores to diagnose coronary artery disease. Clin Cardiol 2002, 25(3):117-22.

10. Griffin BP (ed). Manual of Cardiovascular Medicine. $4^{\text {th }}$ edn. Lippincott Williams \& Wilkins, New Delhi, India; 2013. 02-95p.

11. Kern MJ. The Cardiac Catheterization Handbook. $5^{\text {th }}$ edn, Elsevier, India; 2013. 159-161p.

12. Islam AKMM and Majumder AAS. Coronary artery disease in Bangladesh: A review. Indian Heart J 2013, 65 (4): 424-35.

13. Banerjee SK, Ahmed CM, Rahman MM, Chowdhury MMH and Sayeedet MA. Coronary artery disease in a rural population of Bangladesh: is dyslipidemia or adiposity a significant risk? IMC J Med Sci 2017, 11(2):61-69.

14. Metz CE. Basic principles of ROC analysis. Sem Nuc Med, vol. 8; 1978. 283-298p. 